\title{
Thermal Effects in High-Power End-Pumped Lasers with Elliptical-Mode Geometry
}

\author{
Rüdiger Paschotta, Jürg Aus der Au, and Ursula Keller, Member, IEEE
}

\begin{abstract}
We discuss in detail the effects of thermal lensing and thermally induced stress in end-pumped lasers with a strongly elliptical pump and laser mode and compare this situation with cylindrical rod geometries.
\end{abstract}

Index Terms-High power lasers, laser cavity design, thermal lensing.

\section{INTRODUCTION}

$\mathbf{T}$ HE most common geometry for end-pumped lasers is that of a cylindrical laser rod with circular pump and laser beams propagating along the symmetry axis of the rod. The limitations of this approach are well known: the dissipated power leads to an inhomogeneous temperature distribution in the laser rod and consequently to a tendency for stress fracture as well as for thermal lensing and thermally induced birefringence. The focusing power of the thermal lens varies with pump power and can be strongly aberrative. The latter problem is particularly severe if diffraction-limited operation is required. In the rod geometry, thermal lensing has been reduced to some extent by using rods with undoped ends [1].

In recent years, an alternative geometry for the laser head has been applied to various lasers based on Nd : YAG [2], Cr: LiSAF [3], [4], Nd : glass [5], [6], and Yb: YAG [7]. In most cases, a good beam quality was achieved with rather high output power, typically more than had been achieved with cylindrical rod geometries. A detailed discussion of thermal effects in these lasers has not yet been presented and is the scope of this paper. In Section II, we calculate the maximum temperature rise, thermally induced stress, and thermal lensing. We compare these results to those for the cylindrical rod geometry and discuss further power scaling. The main emphasis is on thermal lensing, while we do not elaborate on thermally induced birefringence. The latter effect is also greatly reduced in the elliptical-mode geometry, in a similar way as discussed in [8] for homogeneously pumped slabs. In our elliptical-mode lasers, it has never led to a significant reduction of polarized output power or beam quality. In Section III, we discuss implications of the cavity design on thermal effects in lasers and outline a strategy to find so-called zone-I cavity designs [9], which are shown to be less sensitive to lensing than the also frequently used zone-II designs.

\footnotetext{
Manuscript received December 13, 1999; revised June 8, 2000. This work was supported by the Swiss Priority Program in Optics.

The authors are with the Institute of Quantum Electronics, Swiss Federal Institute of Technology, Zurich CH-8093, Switzerland.

Publisher Item Identifier S 1077-260X(00)07861-8.
}

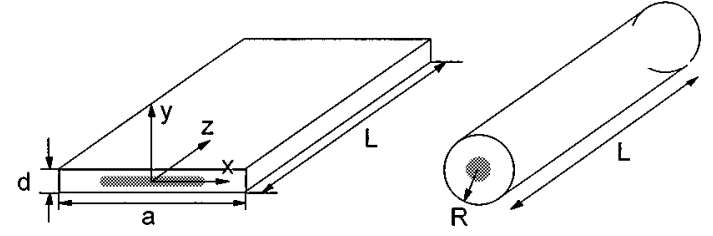

(a)

(b)

Fig. 1. (a) Slab for a laser with elliptical mode geometry. (b) Cylindrical rod geometry.

\section{THEORY}

\section{A. Description of the Geometries}

Fig. 1(a) illustrates the elliptical-mode geometry. The laser medium is a slab of width $a$ (in $x$ direction), thickness $d$ (in $y$ direction), and length $L$ (in $z$ direction). It is cooled from the top and bottom side, and we will always assume that these surfaces are kept at a constant temperature. The pump beam, usually generated with one or two high power diode bars and focused with some cylindrical optics, is injected into the $z=0$ face. In the $y$ direction it will usually have a near-Gaussian profile, while in the $x$ direction the profile can often deviate substantially from a Gaussian function. We will see in Section II-D that this profile has a strong influence on thermal lensing.

For comparison, we also consider the cylindrical rod geometry shown in Fig. 1(b). The rod has a length $L$ and radius $R$, and the pump beam is injected into the $z=0$ face. The end faces are usually not cooled, and we assume the cylindrical rod surface to be kept at constant temperature.

\section{B. Temperature Rise}

Here we consider the maximum temperature rise in the gain medium with respect to the cooled surface. While thermally induced stress and thermal lensing arise from temperature gradients only, the maximum temperature rise determines the operation temperature of the laser medium. For some (typically four-level) laser media this quantity is not of great relevance, but at elevated temperatures the efficiency of three-level laser media like $\mathrm{Yb}$ : YAG is reduced, while some other gain media like $\mathrm{Cr}$ : LiSAF show quenching effects [3], [4], [10]. Such lasers profit immediately from the smaller temperature rise usually achievable with the elliptical-mode geometry.

We first consider the cylindrical rod geometry, for which analytical solutions are readily available [11], [12]. If we assume 
the power $P$ to be dissipated uniformly within a radius $w$ from the rod center (with $w \leq R$ ), we obtain a temperature rise

$$
\Delta T_{\max }=\frac{P}{4 \pi K L}\left(1+2 \ln \frac{R}{w}\right)
$$

of the rod center with respect to the outer surface, where $K$ is the thermal conductivity of the medium. We conclude that for a given material $\Delta T_{\max }$ is largely controlled by the quantity $P / L$, and the only way to substantially decrease $\Delta T_{\max }$ is to increase $L$. Limits to this can be set by the beam divergence (particularly if the pump beam has a poor beam quality), by the availability of long crystals, or by nonlinear effects in pulsed lasers, for example.

Now we consider a slab geometry where for a rough estimate we assume that the pump beam fills the full width $a$ of the slab, that the pump profile is approximately flat in $x$ direction, and that the extension of the beam in $y$ direction is small compared to $d$. This results in the simple equation

$$
\Delta T_{\max }=\frac{P d}{2 K L a} .
$$

We see that for a given material and length $L$, we can decrease $\Delta T_{\max }$ by decreasing the ratio $d / a$. Such an option is not available in the rod geometry. This result is qualitatively still the case if the assumptions made are not strictly valid. As a higher precision is usually not essential for this purpose, we resort on more precise numerical solutions only in the following sections.

\section{Thermally Induced Stress}

The stress that is induced by the inhomogeneous temperature distribution in the gain medium can lead to stress fracture and also have optical effects because of its influence on the refractive index. In this section, we consider only the problem of stress fracture. Optical effects of stress are treated in the next section.

Again we first consider the cylindrical rod geometry, for which analytical solutions are available [11], [12]. As before, we assume the power $P$ to be dissipated uniformly within a radius $w$ (with $w \leq R$ ). The maximum tangential stress at the rod surface is

$$
\sigma_{\max }=\frac{\alpha_{T} E}{4 \pi K(1-\nu)} \frac{P}{L}\left(2-\frac{w^{2}}{R_{2}}\right)
$$

where

$$
\begin{array}{ll}
\alpha_{T} & \text { thermal expansion coefficient; } \\
E & \text { Young's modulus; } \\
\nu & \text { Poisson's ratio. }
\end{array}
$$

As with the maximum temperature rise, we see that for a given material, a significant reduction of stress is possible only by increasing the length $L$.

For the slab geometry, we first discuss the situation in which the slab is uniformly pumped over its whole volume, where [11] gives the equation

$$
\sigma_{\max }=\frac{\alpha_{T} E}{12 K(1-\nu)} \frac{d}{a L} P
$$

for the maximum surface stress. For a given material and given $P / L$, the stress can be reduced by reducing the ratio $d / a$. This is

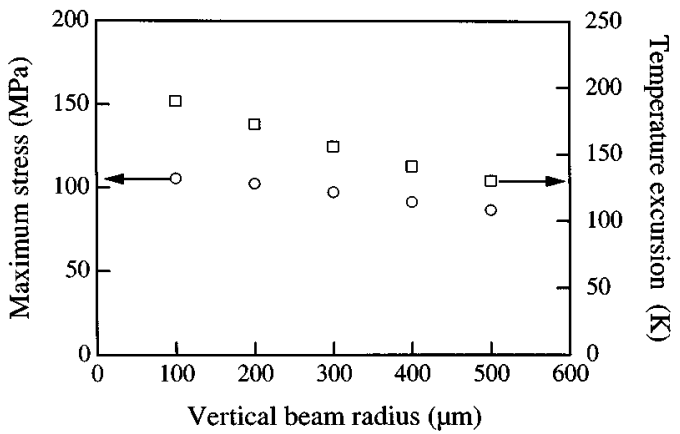

Fig. 2. Calculated maximum temperature rise and maximum stress in a $\mathrm{Nd}$ : glass (LG-760) slab with $L=7.5 \mathrm{~mm}, a=7.8 \mathrm{~mm}, d=1 \mathrm{~mm}$, and $4 \mathrm{~W}$ of dissipated power, where we varied the pump beam width in $y$ direction.

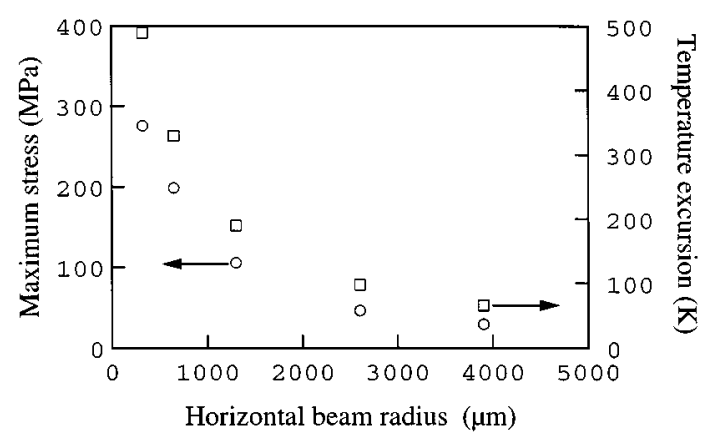

Fig. 3. Calculated maximum temperature rise and maximum stress as in Fig. 2, but with pump beam width in $x$ direction as variable parameter.

remarkable as, e.g., a reduction of $d$ alone leads to an increased density of dissipated power.

For the more difficult situation where only part of the slab is pumped, we used the program SOLIDIS [13] to solve the heat conduction equation numerically. We assumed the pump beam to have the Gaussian radii $w_{x}$ and $w_{y}$ in $x$ and $y$ direction, respectively. First we investigated the dependence of the maximum surface stress on $w_{y}$, while $w_{x}$ and $P$ are kept constant. Fig. 2 shows that the dependence of both $\Delta T_{\max }$ and $\sigma_{\max }$ on $w_{y}$ is weak. Then we varied $w_{x}$ (Fig. 3) and found that $\Delta T_{\max }$ and $\sigma_{\max }$ depend largely on $P / w_{x}$. Thus we conclude that, as in the uniformly pumped slab, the stress depends mainly on $P \cdot d /\left(w_{x} L\right)$ apart from material parameters. To minimize stress fracture, we should therefore use a slab as thin as possible and choose $w_{x}$ large enough. A small value of $w_{y}$ is beneficial because it limits the required magnitude of $d$ and allows to increase $w_{x}$ without making the mode area (and thus the laser threshold) excessively large. The optimum value of $w_{y}$ will often be limited by the divergence of the pump beam. Thus a pump source with good beam quality in $y$ direction can help to decrease both the temperature rise and the stress by using a thinner crystal. High-power diode bars, the currently most powerful pump sources available, happen to have just this quality: while the beam quality factor $M^{2}$ is typically $\gg 1000$ in the horizontal direction, where our demands for a slab geometry are very moderate, we typically have $M^{2}<10$ in the $y$ direction, enabling tight focusing in this direction and thus the use of a rather thin crystal. 


\section{Thermal Lensing}

The inhomogeneous temperature distribution in the gain medium may lead to thermal lensing due to several reasons [11]. The refractive index is directly influenced by the local temperature, and it is also affected by thermally induced stress. Moreover, stress can deform the end surfaces of the gain medium, which gives another contribution to thermal lensing. Which contributions are dominant depends on the gain material as well as on the geometry. Therefore, we will discuss the situation for different lasers.

1) Yb:YAG Laser: We have recently demonstrated a modelocked high-power $\mathrm{Yb}$ : YAG laser based on the elliptical-mode geometry [7]. In two nearly diffraction-limited output beams $\left(M^{2}<1.2\right.$ in sagittal and tangential direction) $8.2 \mathrm{~W}$ of total average power has been obtained. Here we discuss the strong thermal effects in this laser more in detail and derive some more general conclusions from this analysis. First we calculated the temperature and stress distributions and the bulging of the end faces of the $d=1 \mathrm{~mm}$ thick, $a=9 \mathrm{~mm}$ wide, and $L=4$ $\mathrm{mm}$ long $\mathrm{Yb}$ : YAG slab for a total dissipated power of $1 \mathrm{~W}$, using the program SOLIDIS [13]. The heating power density was assumed to be proportional to the local pump intensity. The simulation was carried out for different pump intensity distributions. Then we used the obtained data to calculate the refractive index profile, taking into account both temperature and stress effects [8], [11]. Finally we obtained the phase retardation $\varphi(x, y)$ along lines in $z$ direction by simple integration and calculated the thermal lens power in $x$ and $y$ direction from this. The material data were taken from [11], [12], and [14].

For the first simulation, we assumed the pump profile to be Gaussian in $x$ and $y$ direction with $w_{x}=1.2 \mathrm{~mm}$ and $w_{y}=$ $80 \mu \mathrm{m}$ (neglecting divergence along the $z$ direction), centered in $x$ and $y$ direction. In the second and third simulation we assumed super-Gaussian functions $I \propto \exp \left[-2\left|x / w_{x s}\right|^{s}\right]$ in $x$ direction with $s=3$ and $s=6$, respectively, and calculated $w_{x s}$ so that the peak intensity as well as the second moment of the intensity distribution stays the same as before. Experimentally obtained pump profiles, which we generated with a high-power diode bar and cylindrical optics, were characterized with a charge-coupled device camera and found to be close to the super-Gaussian function with $s=6$ near the focus, while an ordinary Gaussian (with $s=2$ ) is a better fit near the crystal ends. The typical experimental situation effectively lies somewhere between $s=2$ and $s=6$. The pump absorption along the $z$ direction with an absorption length of $3 \mathrm{~mm}$ is taken into account. Fig. 4(a), (c), and (e) shows the resulting temperature distributions. They do not perfectly reflect the pump intensity distributions because the crystal thickness $d$ is not much smaller than the pump beam width. The solid curves in Fig. 4(b), (d), and (f) show the resulting values for the local thermal lens focal power $f_{x}^{-1}$ in $x$ direction, calculated (always for a single pass through the crystal) from the second derivative of the phase $\varphi(x, y)$ with respect to $x$. For the Gaussian profile [Fig. 4(b)], $f_{x}^{-1}$ has its maximum $(0.21 / \mathrm{m})$ at the center, while for the super-Gaussian with $s=6$ [Fig. 4(f)] maximum lensing $(0.17 / \mathrm{m})$ occurs in the wings because the temperature profile is quite flat near the center and steeper in the wings. The other curves in Fig. 4(b), (d), and (f) show the different contributions to thermal lensing. In both cases, the effect of stress on the refractive index somewhat counteracts the direct temperature effect and the effect of bulging of the end faces. The most interesting conclusion is that for a pump profile with $s \approx 3$, which is quite realistic for real situations, the resulting values of $f_{x}^{-1}$ are nearly constant over much of the pump beam width. This means that the effect of aberrations can be quite small if only the laser mode size is chosen to be somewhat smaller than the pump mode size, so that the laser mode does not probe the regions of strong aberration in the wings of the pump profile. Indeed, our $\mathrm{Yb}$ : YAG laser was found to have the best beam quality when the laser mode radius in $x$ direction was around $0.9 \mathrm{~mm}$ [7], to be compared with a pump beam radius around $1.2 \mathrm{~mm}$.

The thermal lens focussing power $f_{y}^{-1}$ in $y$ direction (Fig. 5) is significantly stronger, about $1.8 / \mathrm{m}$ in the beam center. It is important, however, to realize that nevertheless thermal lensing in $y$ direction constitutes a much smaller problem than in $x$ direction because the laser mode size in $y$ direction is much smaller, and smaller modes are less sensitive to focusing effects. As shown in [9], the width of the stability ranges of a standing-wave cavity with respect to the thermal lens focussing power $f^{-1}$ is $2 \lambda /\left(\pi w_{0}^{2}\right)$, where $w_{0}$ is the mode radius in the gain medium at the stationary point (minimum) of the stability range. In our case, the width of the stability range in $y$ direction is more than two orders of magnitude larger than in $x$ direction while the thermal lens is only about one order of magnitude stronger. (Another way to explain this dependence on mode size is that a larger mode acquires a higher phase shift in its wings because, e.g., for a not aberrated lens in $x$ direction the phase shift is proportional to $x^{2} / f_{x}$.) Indeed, we found it much easier to obtain a good beam quality in $y$ direction, despite of the larger $f_{y}^{-1}$.

We also found that the thermal behavior of the $\mathrm{Yb}$ : YAG laser was somewhat improved by using a vertical offset of the pump beam from the center position. A simulation revealed that in this asymmetric situation (with a vertical offset of $300 \mu \mathrm{m}$ ) the temperature rise is reduced by one-third and the thermal lens power by one-fifth. The finding indicates that the thickness $d$ could actually be made somewhat smaller without increasing the diffraction loss too much. This should decrease the temperature rise, stress, and the thermal lens power in $x$ direction. We tried this with a reduced crystal thickness of $0.6 \mathrm{~mm}$ but achieved a lower output power, apparently because the reflecting coating was not good enough so close to the edge of the crystal.

2) Nd:Glass Laser: As another example, we consider the case of Nd: glass high-power lasers, as described in [5] and [6]. The dimensions of the gain medium, a slab made of Nd-doped Schott LG-760 phosphate glass, are similar as for the $\mathrm{Yb}$ : YAG laser: $d=1 \mathrm{~mm}, a=7.8 \mathrm{~mm}$, and $L=7.5 \mathrm{~mm}$. We used material data from the manufacturer [15], except for the photoelastic coefficients for which we used data of a similar phosphate glass Q-88 from [14].

Fig. 6(a) shows the temperature distribution in the $\mathrm{Nd}$ : glass gain medium for $1 \mathrm{~W}$ of dissipated power. We assumed a superGaussian pump profile in $x$ direction with $s=6$ and a width of $w_{x s}=1300 \mathrm{~nm}$ (as in the experiment). The temperature rise is much larger than in YAG because of the 21 times poorer 


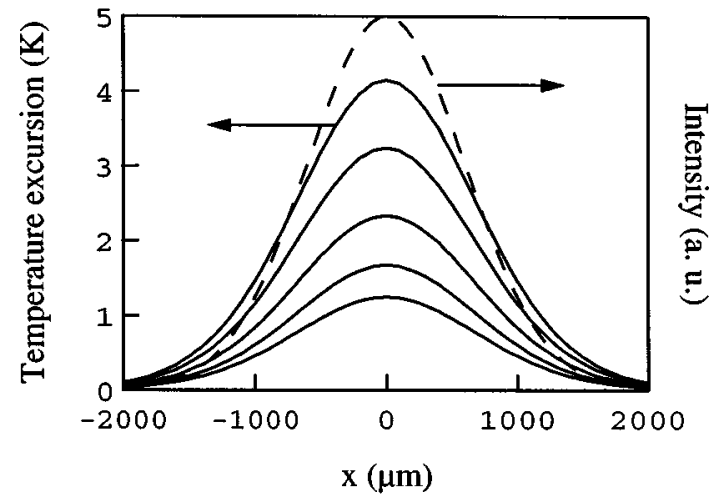

(a)

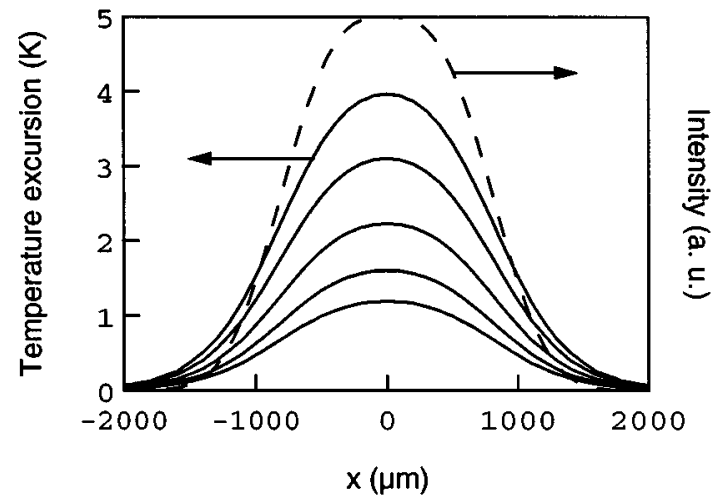

(c)

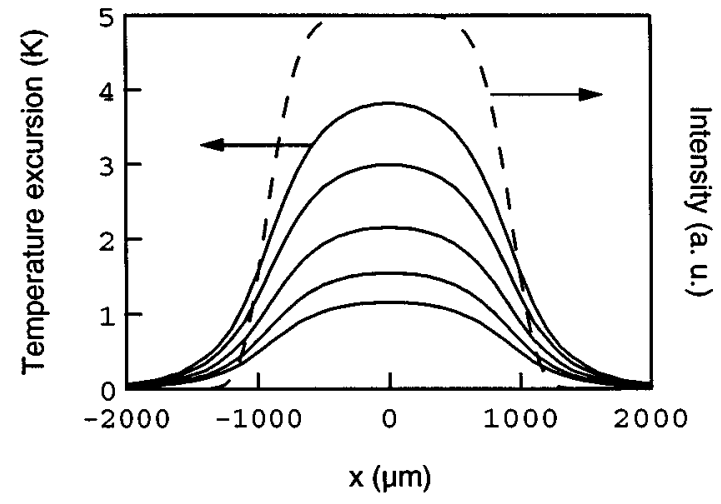

(e)

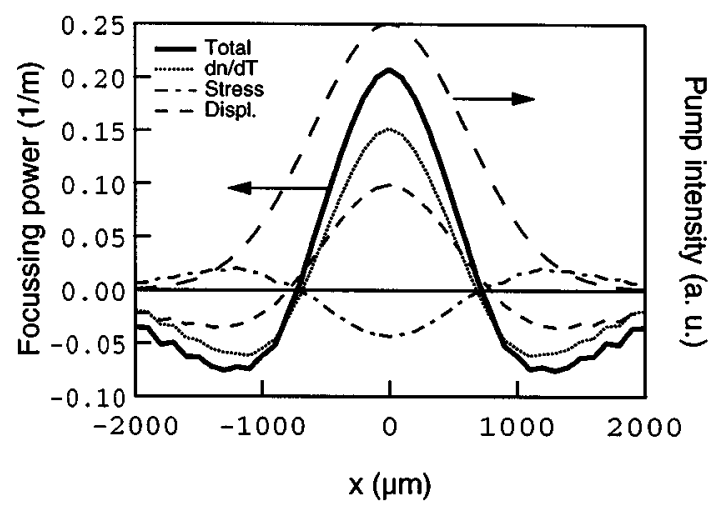

(b)

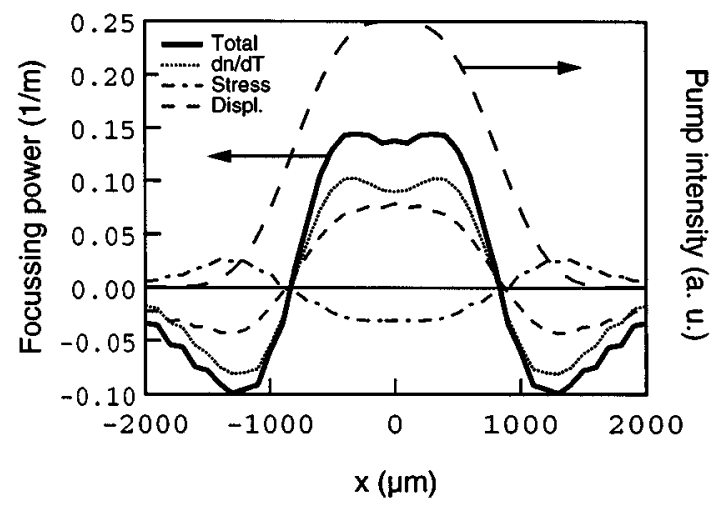

(d)

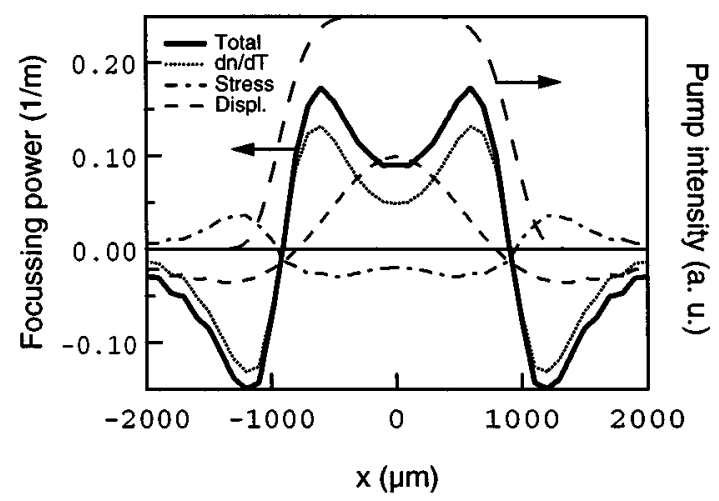

(f)

Fig. 4. (a) Calculated temperature profile in $x$ direction at $y=0 \mathrm{~m}$ and different $z$ positions (upper solid curve: $z=0 \mathrm{~m}$; lower solid curves: $z=1000 \mathrm{~m}$, 2000 $\mathrm{m}, 3000$, and $4000 \mathrm{~m}$, respectively) for a 4-mm-long Yb:YAG slab with $1 \mathrm{~W}$ of absorbed power. The pump profile is Gaussian with $w_{x}=1200 \mu \mathrm{m}, w_{y}=80 \mu \mathrm{m}$. (c) Like (a), but with super-Gaussian profile in $x$ direction (see text) with $s=3$. (e) Like (c), but with $s=6$. (b) Local thermal lens focusing power in $x$ direction for Gaussian pump profile as in (a). Thick solid line: total inverse focal length. Other curves: contributions from the direct thermal effect $(d n / d T)$ on the refractive index, from stress, from bulging of the end faces, and the pump beam profile. (d) and (f) Like (b), but with super-Gaussian profiles $s=3$ and $s=6$, respectively.

thermal conductivity. However, Fig. 6(b) shows that the negative value of $d n / d t$ (a characteristic of the phosphate glass) allows the direct thermal effect on the refractive index to cancel most of the effect caused by stress. Mainly for this reason, the thermal lens focusing power is only four times stronger than in YAG for the same dissipated power. Another factor is that the four-level nature of $\mathrm{Nd}$ : glass allows to work with a somewhat larger mode area, which reduces the effect of thermal lensing (see the next section for details). The main problem is in the end the tendency for stress fracture, which we indeed experienced a few times in our $\mathrm{Nd}$ : glass laser experiments but never with the $\mathrm{Yb}$ : YAG laser, despite of significantly higher output powers.

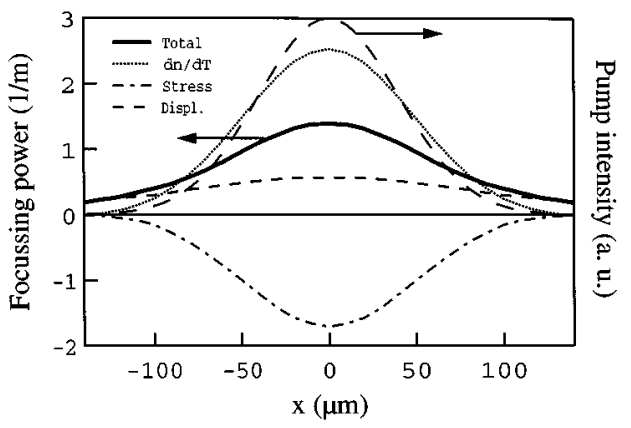

Fig. 5. Like Fig. 4(b), but for the $y$ direction. 


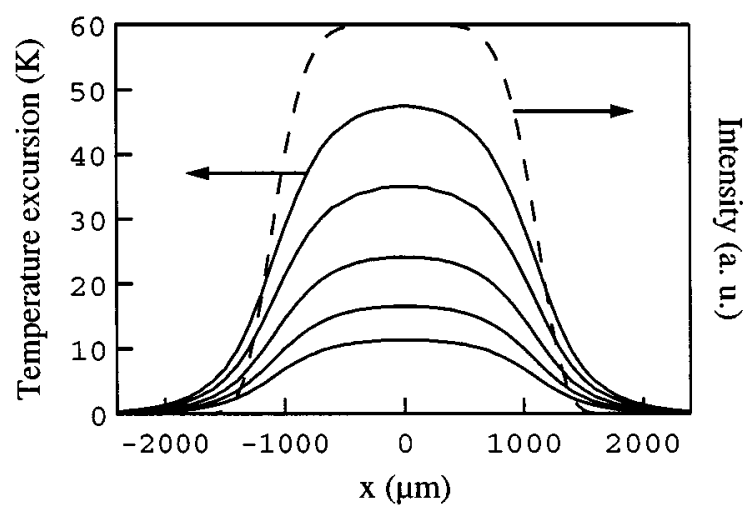

(a)

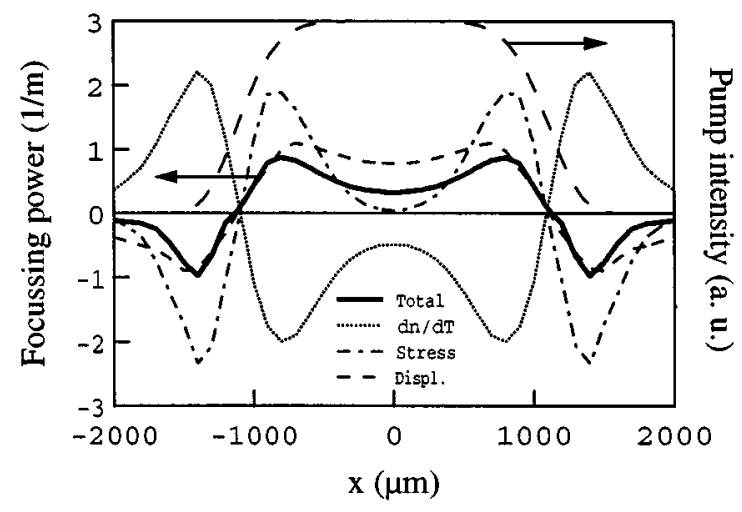

(b)

Fig. 6. Like Fig. 4(e)and Fig. 4(f), respectively, but for Nd: glass and $w_{x s}=1300 \mu \mathrm{m}$.

3) Power Scaling: Scaling to higher powers is possible in the elliptical-mode geometry by increasing the width of the pump and laser beam in the $x$ direction. For the following discussion, we assume the case where the beam width is large compared to the thickness $d$, so that an approximately one-dimensional heat flow is established, and consider only the direct thermal effect on the refractive index (which scales in a similar way to the other effects). If the pump beam width is doubled for a constant power level (without changing the beam shape), while the thickness $d$ is kept constant, the temperature rise is reduced by a factor of two, while the second derivative of the phase $\varphi(x, y)$ with respect to $x$, as well as the thermal lens focusing power $f_{x}^{-1}$, is reduced by a factor of eight. At the same time, however, the beam becomes four times more sensitive to lensing (see the discussion above). Consequently, there is room for doubling the power level without increasing thermal lensing problems. The same is true for stress fracture. Also, doubling the laser mode area and thus the threshold power is acceptable as the pump power is doubled.

Further reduction of thermal lensing in the $x$ direction could be achieved by controlling the shape of the pump beam more carefully. If the beams of two pump diodes are combined (by polarization coupling, as, e.g., in the Yb:YAG laser [7]), the relative alignment of these beams gives another parameter to be optimized. One may adjust a horizontal offset of the beams so as to minimize the strength and the aberrations of the thermal lens. It is also conceivable that improved pump optics could be used to control the pump beam profile in $x$ direction more precisely in order to minimize aberrations. We note that the ideal pump profile is not rectangular but should rather approximate a function that could be optimized using a numerical analysis as described above.

It is interesting to note that thermal lensing in the ellipticalmode $\mathrm{Yb}$ : YAG laser as discussed above is actually not much weaker than it would be in a rod laser. If we calculate the range of absorbed powers in which a standing-wave resonator is stable, this range is roughly one-third larger than for typical YAG rod lasers. Moreover, the temperature rise is roughly the same as it would be in a rod with the same pump beam area. This is because the crystal thickness was not much smaller than the horizontal beam width. The situation is similar in the discussed Nd: glass laser. We conclude that with the present elliptical-mode lasers we have just started to get into the regime where the elliptical-mode geometry is advantageous. The main advantage of the concept is that further power scaling appears to be possible, unlike in a rod geometry. Also we profit already from reduced birefringence losses.

\section{ReMARKS ON LASER CAVITy DESIGN}

The design of a laser cavity strongly influences the sensitivity of the laser to thermal effects. A central challenge is that the strength of the thermal lens varies with pump power, and to some smaller extent also with the intracavity laser intensity. A standing-wave resonator with a single variable lens has in general two stability zones, called zone I and zone II [9]. Stable cavity modes exist if the focusing power of the thermal lens lies in one of these zones. The minimum fundamental mode radius $w_{0}$ is the same in both zones, and the width of both zones in terms of focusing power is $2 \lambda /\left(\pi w_{0}^{2}\right)$. For stable operation with good beam quality, it is advantageous to operate the laser near a minimum of mode size in the laser medium, because then the spot size is less sensitive to changes of the power level. Moreover, it was shown [9] that the alignment of the cavity is significantly more critical in zone II, which is defined as the zone in which the mode sizes on both end mirrors of the cavity diverge at one of the stability limits.

In our experiments on elliptical-mode Yb: YAG lasers, we initially used zone-II cavity designs, but then we found that the alignment stability was indeed greatly improved by changing from a zone-II cavity to another one operating in zone I [7]. We believe that alignment sensitivity is particularly important in lasers were the thermal lens is strong and has significant aberrations. If, for example, the pump beam is slightly moved in transverse direction, this moves the center of the thermal lens away from the beam axis and thus has a similar effect as a tilt of a mirror. If the laser mode reacts to this with a strong change of mode position (which can in turn again influence the strength and shape of the thermal lens), the alignment becomes very critical, even if good mechanical mounts are used. With the initial zone-II cavities, we sometimes observed hysteresis effects, where, for example, the laser power could not be reproduced 


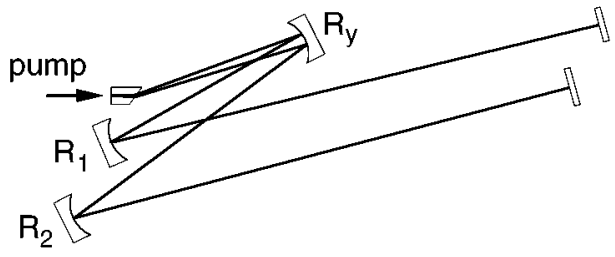

(a)

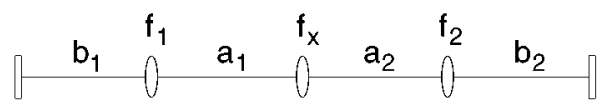

(b)

Fig. 7. (a) Laser cavity for elliptical-mode lasers. The Brewster-angled slab is pumped through a mirror coating on the left side. $R_{y}$ indicates a cylindrical mirror for focusing in $y$ direction. (b) Schematic for the laser cavity in (a), for the $x$ direction only. $f_{x}$ indicates the thermal lens, and $f_{1}$ and $f_{2}$ represent the focal lengths of mirrors $R_{1}$ and $R_{2}$.

after just blocking the pump beam for a moment. This tendency, which makes the alignment very difficult, was found to be much reduced with a zone-I cavity design.

The question then arises how to find a suitable cavity design operating in zone I. This is indeed a difficult task because several conditions have to be fulfilled. The laser mode sizes in the gain medium have to have given values in both directions, and particularly in $x$ direction the operating point should be not too far from the point of minimum mode size. Sometimes, the mode sizes at other positions have to meet additional conditions, e.g., to get the right mode area on a saturable absorber in a mode-locked laser. We note that if a zone-II design is known that meets all these requirements, it cannot be easily transformed into a zone-I design by continuous adjustment of the parameters, because both zones are separated by an unstable region, and there are singularities at the ends of the stability zones.

In this situation we have developed a strategy to find a zone-I design meeting all mentioned requirements. The implementation of this strategy led to a self-made computer program, which is based on a combination of analytical results with a numerical optimization algorithm. In the following, we describe the basic ideas. The type of laser cavity we considered is shown in Fig. 7(a). The gain medium has a highly reflective coating for the laser wavelength on one side, through which the pump power is applied. For two reasons, the laser cavity must be folded at this point. First of all, it can be shown that a standing-wave cavity with the thermal lens at one end always has to operate in zone II, because zone I requires an arbitrarily strong thermal lens if one arm length goes to zero. Also, a cavity with two arms is more suitable for a mode-locked laser because it gives us the freedom to put the output coupler at one end (generating a single output beam) and the saturable absorber on the other end (which makes focussing on the absorber more convenient). A disadvantage of this cavity type is that the beam makes four instead of two passes through the gain medium per round-trip, doubling the effective strength of the thermal lens (but also the gain). The small mode size in $y$ direction is generated by a cylindrical mirror $\left[R_{y}\right.$ in Fig. 7(a)]. The structure of the cavity in $x$ direction, for which we can ignore the cylindrical mirror, is as shown in Fig. 7(b); it contains two curved mirrors $\left(R_{1}, R_{2}\right)$, represented by lenses with the focal length $f_{1}$ and $f_{2}$, and two flat end mirrors, apart from the thermal lens $\left(f_{x}\right)$.

Considering first only the $x$ direction, we can more or less freely choose four arm lengths for a given set of mirrors $R_{1}$ and $R_{2}$. For an arbitrarily chosen set of the four arm lengths $a_{1}, b_{1}, a_{2}, b_{2}$, one can now use the analytical results of [9] combined with the standard ABCD matrix formalism to calculate the focusing power $f_{x}^{-1}$ of the thermal lens at the stationary point in zone I. For this value of $f_{x}^{-1}$, rather than for the actual value in the experiment, we calculate a figure of merit (FOM). This quantity is defined so that it would be zero for an ideal cavity design and contains penalties for all deviations from the desired mode sizes as well as for the deviation of the calculated value $f_{x}^{-1}$ from its actual value in the experiment. This FOM can now be calculated for any set of the arm lengths, and numerical optimization (starting, e.g., from random initial values) leads to an optimized set of these parameters. Because this strategy may lead to a local optimum that is not as good as the global optimum, we started this process repeatedly with random values for the arm lengths and found that it usually converges to one of a few solutions, so that the global optimum can be found with a quite limited number of trials.

After this, we have to optimize the cavity in $y$ direction. Given the radius $R_{y}$ of the cylindrical mirror, the only free parameter is the position of this mirror. As this is usually not sufficient to meet, e.g., two mode sizes criteria (in our case for the laser medium and a saturable absorber) simultaneously, we do the optimization in $x$ direction repeatedly with different mirrors $R_{1}$ and $R_{2}$ and/or a different spot size requirement in $x$ direction for the saturable absorber. The folding angles on the curved mirrors can also used for the optimization.

We used this strategy successfully for the Yb: YAG laser [7] but also for new designs of the same type for $\mathrm{Nd}$ : glass lasers. It usually leads to good solutions because the number of adjustable parameters is larger than the number of requirements to be met, although the situation would be too complicated for a pure trial-and-error method to work within reasonable time. We expect that the basic idea of our design strategy will be useful also for other cases where a design operating in a specific stability zone is desired.

\section{CONCLUSION}

We have discussed in detail thermal effects, particularly stress and thermal lensing, in high-power lasers with an elliptical pump beam and laser mode. We used analytical arguments to discuss how this geometry, in contrast to the conventional rod geometry, allows scaling to higher powers. These arguments are strictly valid only if the pump beam width is large compared to the slab thickness. For some practical situations in which this approximation is not well fulfilled, we carried out numerical simulations from which we could draw a variety of conclusions. The thermal lens in the horizontal direction is weaker than for the vertical direction, but nevertheless more important for beam quality. While in elliptical-mode lasers demonstrated so far the strength of the thermal lens is not much weaker than it would be in a rod geometry, further power scaling will lead 
the designs into a regime where the advantages compared to rods become very significant. Already, thermal degradations of the mode profile are significantly reduced: the pump intensity profile, which is typically obtained from high-power diode bars, was found to lead to quite small aberrations of the thermal lens over a reasonably large range, explaining the good beam quality achieved with overpumping (i.e., with a somewhat reduced laser mode size) in previously demonstrated lasers. For an $\mathrm{Nd}$ : glass laser based on phosphate glass, we found the thermal lens to be weaker than expected from the poor thermal conductivity because the negative value of $d n / d t$ leads to some cancellation of thermal effects.

Finally, we discussed the influence of the cavity design on thermal effects in high-power lasers, in particular the benefits of zone-I cavities, and described a systematic strategy to find such designs for elliptical mode lasers.

\section{REFERENCES}

[1] M. Tsunekane, N. Taguchi, T. Kasamatsu, and H. Inaba, "Analytical and experimental studies on the characteristics of composite solid-state laser rods in diode-end pumped geometry," IEEE J. Select Topics Quantum Electron., vol. 3, pp. 9-18, 1997.

[2] D. C. Shannon and R. W. Wallace, "High-power Nd: YAG laser end pumped by a cw, $10 \mathrm{~mm} \times 1 \mu \mathrm{m}$ aperture, $10-\mathrm{W}$ laser-diode bar," Opt. Lett., vol. 16, pp. 318-320, 1991

[3] D. Kopf, J. Aus der Au, U. Keller, G. L. Bona, and P. Roentgen, "A 400-mW continous-wave diode-pumped $\mathrm{Cr}$ : LiSAF laser based on a power-scalable concept," Opt. Lett., vol. 20, pp. 1782-1784, 1995.

[4] D. Kopf, U. Keller, M. A. Emanuel, R. J. Beach, and J. A. Skidmore, "1.1-W cw Cr: LiSAF laser pumped by a 1-cm diode-array," Opt. Lett., vol. 22, pp. 99-101, 1997.

[5] J. Aus der Au, F. H. Loesel, F. Morier-Genoud, M. Moser, and U. Keller, "Femtosecond diode-pumped Nd: glass laser with more than 1-W average output power," Opt. Lett., vol. 23, pp. 271-273, 1998.

[6] R. Paschotta, J. Aus der Au, G. J. Spühler, F. Morier-Genoud, R. H. Vel, M. Moser, S. Erhard, M. Karszewski, A. Giesen, and U. Keller, "Diodepumped passively mode-locked lasers with high average power," Appl. Phys. B., vol. 70, pp. S25-S31, 2000.

[7] J. Aus der Au, S. F. Schaer, R. Paschotta, C. Hönninger, U. Keller, and M. Moser, "High-power diode-pumped passively modelocked Yb : YAG lasers," Opt. Lett., vol. 24, pp. 1281-1283, 1999.

[8] J. M. Eggleston, T. J. Kane, K. Kuhn, J. Unternahrer, and R. L. Byer, "The slab geometry laser-Part I: Theory," IEEE J. Quantum Electron., vol. 20, pp. 289-301, 1984.

[9] V. Magni, "Multielement stable resonators containing a variable lens," J. Opt. Soc. Amer. A, vol. 4, pp. 1962-1969, 1987.

[10] F. Falcoz, K. Kerboull, F. Druon, F. Balembois, P. Georges, and A. Brun, "Small-signal gain investigations for a continous-wave diode-pumped $Q$-switched Cr: LiSAF laser," Opt. Lett., vol. 21, pp. 1253-1255, 1996.

[11] W. Koechner, Solid-State Laser Engineering, A. L. Schawlow, A. E. Siegman, T. Tamir, and H. K. V. Lotsch, Eds. Heidelberg, Germany: Springer-Verlag, 1996, vol. 1. Springer Series in Optical Sciences.

[12] L. Yan and C. H. Lee, "Thermal effects in end-pumped Nd:phosphate glasses,” J. Appl. Phys., vol. 75, pp. 1286-1292, 1994.

[13] J. Funk, J. G. Korvink, J. Buehler, M. Baechtold, and H. Baltes, "SOLIDIS: A tool for microactuator simulation in 3-D," IEEE J. Microelectromech. Sys., vol. 6, pp. 70-82, 1997.
[14] T. J. Kane, J. M. Eggleston, and R. L. Byer, "The slab geometry laser-Part II: Thermal effects in a finite slab," IEEE J. Quantum Electron., vol. 21, pp. 1195-1210, 1985.

[15] Schott Glass Technologies, Glass for Laser Applications, Data Sheets, 1999.

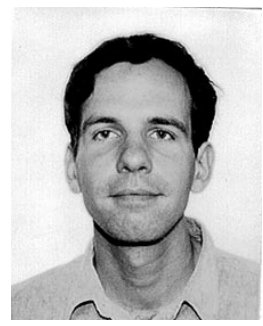

Rüdiger Paschotta was born in Tailfingen, Germany, in 1965. He received the Ph.D. degree from the University of Konstanz, Germany, in 1994 for achievements in the fields of nonlinear optics and quantum optics.

From 1994 to 1997, he was working on fiber lasers and amplifiers at the Optoelectronics Research Centre at the University of Southampton, U.K. In 1997, he moved to the University of Paderborn, Germany, to work in nonlinear optics and integrated optics, and later to the Swiss Federal Institute of Technology (ETH) in Zürich, Switzerland. His research interests are currently focused on all-solid-state picosecond and femtosecond lasers.

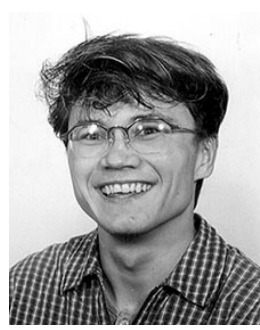

Jürg Aus der Au received the diploma degree in physics from the Swiss Federal Institute of Technology (ETH), Zürich, Switzerland, in 1996, where he is pursuing the Ph.D. degree.

His main research interests are focused on compact high-power femtosecond laser sources.

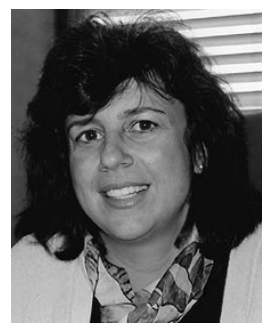

Ursula Keller (M'89) was born in Zug, Switzerland, in June 1959. She received the Diplom in physics from the Federal Institute of Technology (ETH), Zürich, Switzerland, in 1984 and the M.S. and Ph.D. degrees in applied physics from Stanford University, Stanford, CA, in 1987 and 1989, respectively.

From late 1984 to 1985 she worked on optical bistability at Heriot-Watt University, Edinburgh, Scotland. Her Ph.D. research was in optical probing of charge and voltage in GaAs integrated circuits and in low-noise ultrafast laser systems. In 1989, she became a Member of Technical Staff at AT\&T Bell Laboratories, Holmdel, NJ, where she conducted research on photonic switching, ultrafast laser systems, and semiconductor spectroscopy. In 1993, she was appointed an Associate Professor and in 1997 she became a full Professor in the Physics Department of ETH. Her current research interests are in ultrafast lasers, attosecond science, spectroscopy, local probes, and novel devices for applications in optical information processing, communication and medicine. She has published more than 140 journal papers and four book chapters. She holds or has applied for ten patents.

Prof. Keller is a member of the Optical Society of America, the European Physical Society, the Swiss Physical Society, and the Swiss Academy of Technical Sciences. She received a Fulbright Fellowship and was an IBM Predoctoral Fellow. In 1998, she received the Carl Zeiss Research Award for her pioneering work in novel modelocking and $Q$-switching techniques using semiconductor saturable absorber mirrors (SESAMs). 